\title{
Agroturismo Como Modelo De Negocio Para Dinamizar La Economía Familiar De Pequeños Agricultores De Alausí Y Cumandá De La Provincia De Chimborazo
}

\author{
Carla Sofía Arguello Guadalupe \\ Armando Esteban Espinoza Espinoza
}

Docente- Investigador de la Facultad de Recursos Naturales

ESCUELA SUPERIOR POLITÉCNICA DE CHIMBORAZO, Ecuador

Marcelo Eduardo Sánchez, Salazar

William Patricio Cevallos Silva

Docente- Investigador de la Facultad de Administración de Empresas

ESCUELA SUPERIOR POLITÉCNICA DE CHIMBORAZO, Ecuador

Carmen Cecilia Mejía Calle

Docente- Investigador del Centro de Idiomas

ESCUELA SUPERIOR POLITÉCNICA DE CHIMBORAZO, Ecuador

Myriam Isabel Piray Quezada

Investigador- Externo

Carla Jakeline Buestan Carrera

Estudiante- Facultad de Recursos Naturales

ESCUELA SUPERIOR POLITÉCNICA DE CHIMBORAZO, Ecuador

Doi: 10.19044/esj.2017.v13n32p187 URL:http://dx.doi.org/10.19044/esj.2017.v13n32p187

Abstract

This research work is a proposal of a model for an inclusive Agritourism business which has been created in order to invigorate the family economy of marginal farmers in Cumanda and Alausi - towns in the Chimborazo Province, Ecuador. Once the tourist potential in the zone, the one which is linked to the farming production systems, was evaluated, an agritourism product was designed for each one of the towns mentioned, and finally, the model Canvas was applied to the proposal. The research combines field, analytic, and participative methods along with techniques such as the interview and the survey. In order to evaluate the tourist potential in the zone, the MINTUR (2004) tourist attractions inventory methodology was applied. These attractions would later be validated in a participative way along with the community with whom the tourist route would also be created. All this is summarized through the business model proposed by Canvas. Thirty- three 
economically relevant-for-the-population farming products were identified. A tourist route was developed, and it integrated the production systems which have a hierarchy II and III. Moreover, the private and communitarian tourist plant was also designed. The business model proposes the creation of an economically solidary enterprise as a strategy for sustainable development.

Keywords: Agro Tourism, Canvas Business Model, Alausí, Cumanda, Chimborazo

\section{Resumen}

La investigación propone un modelo de negocio inclusivo basado en el agroturismo, para dinamizar la economía familiar de los pequeños agricultores en los cantones de Cumandá y Alausí de la provincia de Chimborazo. Para ello una vez evaluado el potencial turístico de la zona vinculados a los sistemas de producción agropecuarios, se diseña un producto de agroturismo para los dos cantones y finalmente se aplica el modelo Canvas a la propuesta. La investigación combina métodos de campo, analítico, participativo e investigativo, acompañado de técnicas como la encuesta y entrevista. Para evaluar el potencial turístico de la zona se aplica la metodología de inventarios de atractivos turísticos del MINTUR (2004). Atractivos que posteriormente son validados en forma participativa con la comunidad y además se construye la ruta turística. Lo que se resume a través del modelo de negocio propuesto por Canvas. Se identificaron 33 productos agropecuarios de relevancia económica para la población, se desarrolló una ruta turística que integra los sistemas de producción, atractivos de jerarquía II y III además de la planta turística privada y comunitaria. El modelo de negocio propone la creación de una empresa de economía solidaria, como estrategia y propuesta de desarrollo sostenible.

Palabras-claves: Agroturismo, Modelo de negocio Canvas, Alausí, Cumanda, Chimborazo

\section{Introducción}

Las creación de nuevas rutas turísticas en el Ecuador, se ha convertidos en una oportunidad para las comunidades que buscan mejorar sus ingresos, aprovechando esa variedad de atractivos naturales, culturales y en este caso particular agrícolas. La provincia de Chimborazo basa su economía principalmente en la actividad agropecuaria, la zona centro del país es uno de los principales abastecedores de cereales, leguminosas, hortalizas y tubérculos, tanto para la sierra como para la costa. E1 54\% de la población que vive en el sector rural posee pequeñas extensiones con variedad de cultivos (Banco Central del Ecuador, 2012: 1). 
El sistema que integra las nuevas carteras del Estado ecuatoriano como el MAGAP (Ministerio de Agricultura, Ganadería, Acuacultura y Pesca) y MINTUR (Ministerio de Turismo) han identificado como una potencialidad a desarrollar el agroturismo. En base a esto la provincia de Chimborazo en los cantones Alausí y Cumandá, cuenta con una variedad de recurso que pueden ser aprovechados de manera sustentable, buscando generar empleo, equidad, igualdad y mayores oportunidades para los pobladores de sectores rurales (Mintur, 2007: 14).

La actividad agropecuaria en la provincia ha ido disminuyendo con el pasar de los años, esto principalmente debido a la falta de motivación del agricultor, por continuar con sus cultivos, las razones se sustentan en el precio de los productos en el mercado, la alta presencia de intermediarios, la migración de la población del campo a la ciudad, entre otras. El Instituto de Estadísticas y Censos ubica a la provincia de Chimborazo en el puesto número 14 de las provincial del país que destina su territorio a producción agrícola. Dedicando 2.734 ha a la producción de cultivos permanentes y 41.949 ha cultivos transitorios. Esto sin duda afecta a la zona centro del país, disminuyendo la posibilidad de mejorar los ingresos de los pequeños agricultores (Inec, 2014:15).

Es por ello que hoy en día es oportuno aprovechar los recursos agrícolas para vincularlos con las actividades turísticas a través de rutas. Alrededor del mundo se han generado nichos de mercado que viajan constantemente a través de productos de agroturismo. La publicación Gastronomic Sciences, (2008) sobre las rutas alimentarias, menciona que éstas son consideradas como una arquitectura turística basada en la identidad de los alimentos. Productos de este tipo han incidido en el mejoramiento de las condiciones de producción, la revalorización de los saberes locales y la puesta en valor de no solo la actividad agrícola como sustento familiar, sino, como producto turístico, que procura la consecución de una experiencia de viaje distinta a otras que se encuentran en el mercado (Vittorio, 2012-2013: 3).

En este sentido, la creación de una ruta de agroturismo puede establecerse como un sistema económico, social, solidario y sostenible que involucra la participación de la población, proponiendo cambios en el ciclo económico y estableciendo políticas para impulsar el desarrollo soberano de los sectores estratégicos de la provincia y el país, sin dejar de lado la responsabilidad social de los recursos. El cambio de la matriz productiva es la redefinición de la composición de la oferta de bienes y servicios, orientada hacia la diversificación productiva basada en la incorporación de valor agregado, en el impulso a las exportaciones y su expansión en productos y destinos, en la sustitución de importaciones (Senplades, 2013- 2017: 73).

Para lograr consolidar una empresa es necesario diseñar el modelo de negocio, al trabajar en el sector rural la mayoría de la población es indígenas, 
lo que significa que se necesita una herramienta fácil y gráfica para lograr una mayor comprensión de los involucrados, por ello es apropiado aplicar el modelo Canvas. Propuesta por Alex Osterwalder, quien desarrollo una herramienta sencilla y visual que permite diseñar, reflexionar y mejorar aspectos más relevantes para la puesta en marcha de un negocio. La mayor ventaja de este modelo es que puede ser construido con la población paso a paso e involucra a cada uno de los componentes del proyecto que en el futuro serán implementados por los pequeños agricultores de los cantones Cumandá y Alausí (Buestán, 2015:12).

Cuando un emprendedor se plantea una idea por primera vez, no siempre visualiza claramente la forma de hacerlo tangible, de encontrarlo rentable, ya sea en el corto, mediano o en el largo plazo. Así como de saber cuánto dinero requiere para iniciar su idea, de donde obtendrá ese dinero y sobre todo a quiénes debe dirigir su innovación. Ahí surge la necesidad de establecer una forma de proceder, de emplear una metodología, un modelo, es decir, un plan de negocios. El método cavas es una herramienta (lienzo) muy práctica ya que te permite modificar todo lo que quieras sobre la misma a medida que vas avanzando en su análisis y testando las hipótesis más arriesgadas que ponen en juego la viabilidad de tu negocio (Ferreira, 2015:71).

\section{Metodología}

Esta investigación fue de tipo no experimental, utilizó los métodos deductivo, inductivo y analítico para la recolección de datos; además el método participativo pues no solo se validó los atractivos turísticos con los pequeños agricultores, sino además se construyó en forma conjunta la mejor ruta agroturistica. Además los métodos bibliográfica y de campo, se aplicó el análisis crítico y comparativo, procesos que juntos lograron el cumplimiento de los objetivos de la siguiente manera:

\section{Para la Evaluación del potencial turístico vinculado a los sistemas de producción agrícola}

Se aplicó técnicas participativas mediante talleres y se revisaron fuentes bibliográficas secundarias sobre datos agropecuarios en las zonas de intervención. La información obtenida se sistematizó en una ficha técnica propuesta por Varela (2012) y modificada para la investigación. Cada ficha cuenta con los siguientes componentes:

a. Características generales de sistemas de producción agrícola, pecuaria y piscícola: (localización, variedades, datos históricos relevantes y valor nutricional)

b. Características específicas: (formas del sistema y descripción del proceso)

c. Características culturales alrededor de los sistemas. 
Esto fue complementado con el análisis de la realidad del territorio se consideró conveniente realizar un análisis a nivel del sistema turístico basado en los componentes: Atractivos naturales y culturales, infraestructura básica, planta turística, superestructura y demanda. Cada uno de estos componentes utilizó metodologías de observación, análisis y síntesis, acompañados de técnicas e instrumentos de participación como encuestas, entrevistas y análisis de focos group.

El diseño técnico de la ruta consideró cinco componentes: estructura técnica de la ruta, planteamiento de estrategias de mercadeo, estudio legal administrativo, evaluación ambiental y sociocultural, estudio económico y evaluación financiera. Para la Estructura técnica de la ruta se lo hace a través de dos escenarios. El primer utilizó el siguiente procedimiento:

Revisión y análisis del Plan Estratégico de Desarrollo de Turismo Sostenible en Ecuador hacia el año 2020 ( PLANDETUR), para recuperar la misión, visión, políticas, objetivos y estrategias del desarrollo turístico en el país y la región. Recopilación de información secundaria del turismo en la provincia de Chimborazo, a partir de lo cual se identificó, caracterizó y articuló la oferta existente en el territorio, considerando a la además los gustos y preferencias de la demanda.

El segundo escenario, se aplicó la metodología técnica del diseño de la ruta que potencializa el turismo sostenible en la Región Amazónica Ecuatoriana (2008) que contempla los siguientes componentes: 1. Mapa de identificación de la ruta, 2. Objetivo de la ruta: se especificó el objetivo a largo plazo que se persigue alcanzar con la estructuración de la ruta. 3. Concepto de la ruta. 4. Demanda potencial. 5. Potencialidad turística. 6. Planta turística disponible. 7. Infraestructura social básica disponible: se analizó el estado actual de los servicios básicos (agua, energía, accesibilidad, salubridad, comunicación, entre otros) existentes en los sitios de visita y zonas cercanas a la ruta para la prestación de servicios. 8. Actividades turísticas principales y complementarias: se definieron las actividades turísticas principales y complementarias que pueden realizarse en la ruta. 9. Localidades receptoras. 10. Paquetes turísticos. 11. Gestión de la ruta. 12. Requerimientos de la ruta.

b. Elaboración de estrategia de comercialización.- Se propusieron estrategias de comercialización basadas en los componentes del Marketing Mix (plaza, producto, precio, promoción).

c. Elaboración del estudio legal y administrativo del producto.Partiendo de las condiciones expuestas en la Constitución de la República del Ecuador, Ley de Turismo, Reglamento General de Actividades Turísticas del MINTUR (2004) .

d. Evaluación ambiental y sociocultural.- Para medir el impacto ambiental y sociocultural que podría ocasionar la operación de la ruta, se aplicó la matriz de evaluación de Lázaro Lagos donde se identificaron las 
causas y efectos de la actividad turística con la finalidad de evaluar los impactos de tipo biológico, socio cultural, económico y otros para tomar medidas de mitigación de impactos.

e. Determinación de la viabilidad económica.- Se determinaron las inversiones requeridas para el funcionamiento de la ruta de conformidad con el planteamiento establecido en los estudios anteriores. Para determinar la viabilidad económica se trabajó en el análisis de: 1 . Activos fijos, diferidos y capital de trabajo 2. Fuentes de financiamiento y uso de fondos. 3. Cálculo de depreciaciones y amortizaciones. 4. Presupuesto de costos y gastos. 5. Costo e ingresos. 6. Estado de resultado. 7 Flujo de caja.

f. Análisis financiero

Se realizó el análisis financiero para medir la capacidad que tiene la ruta de generar un flujo de caja que le permita cubrir la inversión, para lo cual se calculó el Valor Actual Neto (VAN), la Tasa Interna de Retorno (TIR) y la relación costo - beneficio, obteniendo así valores que determinen si el producto está en la capacidad de producir o no ganancias.

\section{Para la aplicación del modelo Canvas a la propuesta.}

El modelo cavas es una de las herramientas más empleadas en la metodología Lean Startup, sugerida antes de redactar cualquier memoria de proyecto o plan de empresa, explica todos los apartados que componen el modelo de negocio, es crítico esbozar y realizar un análisis para desarrollar el modelo de negocio de una manera rápida y sencilla. La mejor herramienta para realizar este trabajo previo de reflexión y análisis, es el modelo Canvas de tu negocio o Business (Prim, 2014).

El Canvas es una herramienta nueva, que aparece en 2010, gracias al libro "Generación de modelos de negocio" (Business Model Generation) escrito por Alex Osterwalder e Yves Pigneur. Este libro se caracteriza por ser una guía para visionarios, revolucionarios y retadores que quieren desafiar los anticuados modelos de negocio y diseñar las empresas del futuro.

El modelo se divide en nueve módulos, la parte derecha es la que hace referencia a los aspectos externos a la empresa como el mercado, el entorno. La parte derecha del modelo de negocio Canvas se compone de los siguientes bloques: segmento de mercado, propuesta de valor, canales, relación con los clientes y fuentes de ingresos. En la parte izquierda del lienzo de negocios, se reflejan los aspectos internos de la empresa como asociaciones clave, actividades y recursos clave, y estructura de costes.

Cada una de estas partes es de vital importancia para entender tu modelo de negocio, la segmentación de mercado te permitirá focalizar tu clientela (quien son, que necesidades tienes). En base a ello podrás construir tu propuesta de valor es decir que ofreces al mercado, como vas a distribuir tu producto, de que forma el cliente tiene acceso a él, lo que te dará una idea de 
cuáles podrían ser tus posibles ingresos con la venta de tu producto. En la segunda parte el lienzo te hace pensar en tus estrategias, tus nexos, necesidades de recursos y tu estructura de costos con estos ya tienes idea de que necesitas para empezar tu proyecto, cuando recursos necesitas sean estos humanos, tecnológicos y capital. Y sobre todo sabrás si eres o no competitivo en el mercado.

\section{Resultados}

- $\quad$ Potencial turístico vinculado a los sistemas de producción agrícola

Como ya se mencionó para identificar el potencial turístico de la zona se realizó un estudio de mercado basado en dos procesos principales identificación de la oferta y de la demanda esto se resume a continuación:

\section{- Oferta}

Cuadro 1. Características generales de los productos agrícolas del cantón Alausí

\begin{tabular}{|c|c|c|c|}
\hline $\begin{array}{l}\text { PRODUCTOS } \\
\text { PRINCIPALES }\end{array}$ & $\begin{array}{c}\text { NOMBRE } \\
\text { CIENTÍFICO }\end{array}$ & VALOR NUTRICIONAL & $\begin{array}{c}\text { RESEÑA HISTÓRICA DEL } \\
\text { PRODUCTO } \\
\end{array}$ \\
\hline Cebada & $\begin{array}{l}\text { Hordeum } \\
\text { vulgare }\end{array}$ & $\begin{array}{l}\text { La cebada contiene gran } \\
\text { cantidad de vitaminas, } \\
\text { minerales y contiene } \\
\text { aproximadamente unas } 20 \\
\text { enzimas. }\end{array}$ & $\begin{array}{l}\text { La cebada desciende de la } \\
\text { cebada silvestre, la cual crece en } \\
\text { el Oriente Medio. De gran } \\
\text { importancia para el consumo en } \\
\text { el antiguo Egipto. }\end{array}$ \\
\hline Haba & Vicia faba & $\begin{array}{c}\text { Las habas secas aportan } \\
\text { proteínas, similar a la } \\
\text { carne. Las habas frescas } \\
\text { ideal para dieta equilibrada } \\
\text { y nutritiva. } \\
\end{array}$ & $\begin{array}{l}\text { Se trata de uno de los cultivos } \\
\text { más antiguos del mundo, ya que } \\
\text { existen testimonios de su } \\
\text { domesticación en el periodo } \\
\text { Neolítico -6.000 años a.C. }\end{array}$ \\
\hline Papa & $\begin{array}{l}\text { Solana } \\
\text { tuberosum }\end{array}$ & $\begin{array}{l}\text { La papa presenta un alto } \\
\text { contenido de hidratos de } \\
\text { carbono, vitaminas y } \\
\text { minerales. }\end{array}$ & $\begin{array}{c}\text { La papa es originaria de } \\
\text { América es una planta } \\
\text { alimenticia con gran aporte de } \\
\text { carbohidratos, que está presente } \\
\text { desde las culturas Pre - Incas e } \\
\text { Incas. }\end{array}$ \\
\hline Maíz & Zea mays $L$. & $\begin{array}{l}\text { Es rico en vitaminas del } \\
\text { grupo B (B1 y B3 } \\
\text { principalmente), fósforo y } \\
\text { magnesio. }\end{array}$ & $\begin{array}{l}\text { El maíz era un alimento básico } \\
\text { de las culturas indígenas } \\
\text { americanas muchos siglos antes } \\
\text { de que los europeos llegaran a } \\
\text { América. }\end{array}$ \\
\hline Trigo & $\begin{array}{l}\text { Triticum } \\
\text { aestivum L. }\end{array}$ & $\begin{array}{l}\text { El trigo es un alimento } \\
\text { completo, esta composición } \\
\text { por minerales, como el } \\
\text { fósforo, el calcio, el } \\
\text { magnesio y el silicio. }\end{array}$ & $\begin{array}{c}\text { El trigo fue uno de los primeros } \\
\text { productos cultivados a partir de } \\
\text { la revolución neolítica en el } \\
\text { Próximo Oriente, alimento } \\
\text { básico del hombre en el viejo } \\
\text { mundo. }\end{array}$ \\
\hline Chocho & $\begin{array}{l}\text { Lupinus } \\
\text { mutabilis } \\
\text { Sweet }\end{array}$ & $\begin{array}{l}\text { Posee alto contenido de } \\
\text { proteína y grasas, el chocho } \\
\text { es conocido como la soya } \\
\text { andina. }\end{array}$ & $\begin{array}{l}\text { El chocho es una leguminosa } \\
\text { cultivada por los antiguos } \\
\text { pobladores de la región andina } \\
\text { central desde épocas pre- } \\
\text { incaicas } 2200 \text { a } 2500 \text { años a.c, }\end{array}$ \\
\hline
\end{tabular}




\begin{tabular}{|c|c|c|c|}
\hline Arveja & $\begin{array}{l}\text { Pisum } \\
\text { sativum }\end{array}$ & $\begin{array}{l}\text { Las arvejas son ricas en } \\
\text { hidratos de carbono, y } \\
\text { fibra, contiene más } \\
\text { proteínas que las hortalizas. } \\
\text { Rica en vitamina y } \\
\text { minerales. }\end{array}$ & $\begin{array}{l}\text { Conocido como guisante o } \\
\text { chícharo. La arveja es un } \\
\text { alimento oriundo de Oriente } \\
\text { Medio y de Asia Central. }\end{array}$ \\
\hline Lenteja & $\begin{array}{c}\text { Lens } \\
\text { culinaris }\end{array}$ & $\begin{array}{l}\text { Alto contenido de proteínas } \\
\text { y minerales como calcio, } \\
\text { hierro, magnesio, zinc, } \\
\text { fósforo y vitaminas. }\end{array}$ & $\begin{array}{l}\text { Los primeros indicios del cultivo } \\
\text { datan de unos } 7000 \text { a } 9000 \text { años } \\
\text { de antigüedad. La civilización } \\
\text { egipcia fueron los primeros } \\
\text { exportadores de lentejas de la } \\
\text { antigüedad. }\end{array}$ \\
\hline Fréjol & $\begin{array}{l}\text { Phaseolus } \\
\text { Vulgaris L. }\end{array}$ & $\begin{array}{l}\text { Posee un alto contenido de } \\
\text { hierro, ayuda a corregir } \\
\text { desórdenes biliares, } \\
\text { enfermedades reumáticas, } \\
\text { disminuye la tasa de } \\
\text { colesterol y es eficaz contra } \\
\text { la anemia. Contiene gran } \\
\text { cantidad de vitaminas y } \\
\text { minerales. }\end{array}$ & $\begin{array}{l}\text { Se encuentran entre las primeras } \\
\text { plantas alimenticias } \\
\text { domesticadas y luego cultivadas. } \\
\text { El fréjol domesticado más } \\
\text { antiguo se ha encontrado en la } \\
\text { cueva del Guitarrero en Perú. En } \\
\text { México que se encuentra en } \\
7000 \text { A.C. }\end{array}$ \\
\hline Chirimoya & $\begin{array}{l}\text { Annona } \\
\text { Cherimola }\end{array}$ & $\begin{array}{l}\text { Contiene nutrientes, } \\
\text { hidratos de carbono, } \\
\text { minerales, vitaminas, } \\
\text { antioxidantes y ácidos } \\
\text { grasos. } \\
\end{array}$ & $\begin{array}{l}\text { La chirimoya tiene su origen en } \\
\text { la cordillera de Los Andes (Perú, } \\
\text { Ecuador, Colombia y Chile). Los } \\
\text { conquistadores españoles la } \\
\text { denominaron "manjar blanco" }\end{array}$ \\
\hline Tomate riñón & $\begin{array}{l}\text { Lycopersicum } \\
\text { esculentum }\end{array}$ & $\begin{array}{l}\text { Los principios activos más } \\
\text { importantes del tomate son } \\
\text { las vitaminas A, B y C. }\end{array}$ & $\begin{array}{l}\text { Su origen es del Suroeste de } \\
\text { América. El tomate fue } \\
\text { introducido en Europa por los } \\
\text { colonizadores. }\end{array}$ \\
\hline Aguacate & $\begin{array}{l}\text { Persea } \\
\text { americana }\end{array}$ & $\begin{array}{c}\text { Se destaca en su alto } \\
\text { contenido en Vitamina E, } \\
\text { poderoso antioxidante } \\
\text { natural, que reduce el } \\
\text { riesgo a padecer } \\
\text { enfermedades } \\
\text { cardiovasculares y otras } \\
\text { degenerativas como el } \\
\text { cáncer. } \\
\end{array}$ & $\begin{array}{c}\text { El aguacate es un producto } \\
\text { originario del Continente } \\
\text { Americano, donde los Aztecas le } \\
\text { conferían propiedades } \\
\text { afrodisíacas. Conocido bajo el } \\
\text { nombre de "ahuacatl". Ya en el } \\
\text { siglo XVII los españoles, que le } \\
\text { otorgaron la denominación de } \\
\text { Pera de las Indias } \\
\end{array}$ \\
\hline
\end{tabular}

Cuadro 2. Características generales de los productos agrícolas del cantón Cumandá

\begin{tabular}{|c|c|c|c|}
\hline $\begin{array}{c}\text { PRODUCTO } \\
\text { PRINCIPAL }\end{array}$ & $\begin{array}{c}\text { NOMBRE } \\
\text { CIENTÍFICO }\end{array}$ & VALOR NUTRICIONAL & RESEÑA HISTÓRICA \\
\hline Cacao & $\begin{array}{c}\text { Theobroma } \\
\text { cacao L }\end{array}$ & $\begin{array}{c}\text { El cacao aporta vitaminas } \\
\text { A y y minerales como el } \\
\text { calcio, fósforo, hierro, } \\
\text { magnesio, cobre y potasio. }\end{array}$ & $\begin{array}{c}\text { Utilizada desde las culturas } \\
\text { mesoamericanas, incluso llegó a } \\
\text { usarse como moneda de los } \\
\text { aztecas quienes también atribuían } \\
\text { el origen del cacao a un regalo de } \\
\text { Quetzalcoatl. Los mayas, a su } \\
\text { vez, lo atribuían a Kukulkan, la } \\
\text { misma deidad. }\end{array}$ \\
\hline Maíz & Zea mays $L$. & $\begin{array}{c}\text { Es rico en vitaminas del } \\
\text { grupo B (B1 y B3 } \\
\text { principalmente), fósforo y } \\
\text { magnesio. }\end{array}$ & $\begin{array}{c}\text { En las civilizaciones Maya y } \\
\text { Azteca jugó un papel } \\
\text { fundamental en las creencias }\end{array}$ \\
\hline
\end{tabular}




\begin{tabular}{|c|c|c|c|}
\hline $\begin{array}{l}\text { PRODUCTO } \\
\text { PRINCIPAL }\end{array}$ & $\begin{array}{c}\text { NOMBRE } \\
\text { CIENTÍFICO }\end{array}$ & VALOR NUTRICIONAL & RESEÑA HISTÓRICA \\
\hline & & & $\begin{array}{l}\text { religiosas, en sus festividades y } \\
\text { en su nutrición. }\end{array}$ \\
\hline $\begin{array}{l}\text { Caña de } \\
\text { azúcar }\end{array}$ & $\begin{array}{l}\text { Saccharum } \\
\text { officinarum }\end{array}$ & $\begin{array}{l}\text { Contiene glucosa y fructosa } \\
\text { en menor medida. Posee } \\
\text { cantidades apreciables de } \\
\text { vitaminas A, B, C, D y E. } \\
\text { Y gran cantidad de } \\
\text { minerales. }\end{array}$ & $\begin{array}{l}\text { La caña de azúcar fue trasladada } \\
\text { a España por los árabes en el } \\
\text { siglo VIII A.C. Posteriormente } \\
\text { los españoles llevaron la planta a } \\
\text { las Indias Occidentales. }\end{array}$ \\
\hline Fréjol & $\begin{array}{l}\text { Phaseolus } \\
\text { Vulgaris L. }\end{array}$ & $\begin{array}{l}\text { Posee un alto contenido de } \\
\text { hierro, ayuda a corregir } \\
\text { desórdenes biliares, } \\
\text { enfermedades reumáticas, } \\
\text { disminuye la tasa de } \\
\text { colesterol y es eficaz contra } \\
\text { la anemia. }\end{array}$ & $\begin{array}{l}\text { El frejol domesticado más } \\
\text { antiguo se ha encontrado en la } \\
\text { Cueva del Guitarrero, un sitio } \\
\text { arqueológico en Perú, y se ha } \\
\text { aproximado su fecha alrededor } \\
\text { del segundo milenio a.C. }\end{array}$ \\
\hline $\begin{array}{c}\text { Banano } \\
\text { (orito) }\end{array}$ & $\begin{array}{c}\text { Musa } \\
\text { acuminata }\end{array}$ & $\begin{array}{l}\text { El orito al igual que los } \\
\text { demás bananos, es una } \\
\text { excelente fuente de } \\
\text { vitamina B6 (importante } \\
\text { para la salud del corazón), } \\
\text { vitamina C, fibra y potasio. } \\
\text { A partir de } 1940 .\end{array}$ & $\begin{array}{l}\text { El banano (orito) es una fruta } \\
\text { originaria del sur de Asia. Inicio } \\
\text { en Ecuador en 1940. En la } \\
\text { década de los años } 50 \text { se dio el } \\
\text { boom bananero convirtiéndose el } \\
\text { Ecuador en el primer exportador } \\
\text { mundial de la fruta }\end{array}$ \\
\hline
\end{tabular}

Otros atractivos agroturísticos lo componen los productos pecuarios como: Bovinos, ovinos, camélidos, porcinos, equinos, aves de corral, cuyes presentes en ambos cantones. Además es posible encontrar productos piscícolas como Truchas y Tilapias que el turista puede por sí solo pescar y disfrutar de la naturaleza y su entorno. Es también parte de la oferta los atractivos naturales resumidos en la siguiente cuadro 3 .

Cuadro 3. Matriz de sistematización de atractivos turísticos

\begin{tabular}{|c|c|c|c|}
\hline \multicolumn{4}{|c|}{ MATRIZ DE SISTEMATIZACIÓN DE ATRACTIVOS TURÍSTICOS } \\
\hline \multicolumn{4}{|c|}{ CLASIFICACIÓN } \\
\hline & CATEGORIZACIÓN & $\begin{array}{l}\text { CANTIDAD } \\
\text { DE } \\
\text { ATRACTIVO } \\
\text { S }\end{array}$ & $\begin{array}{l}\text { PORCENTAJ } \\
\text { E }\end{array}$ \\
\hline \multirow{13}{*}{ CLASIFICACIÓN } & SITIOS NATURALES & 10 & 38 \\
\hline & $\begin{array}{l}\text { MANIFESTACIONES } \\
\text { CULTURALES }\end{array}$ & 16 & 62 \\
\hline & TIPO & & \\
\hline & Ambientes lacustres & 2 & 8 \\
\hline & Montaña & 1 & 4 \\
\hline & Bosque & 2 & 8 \\
\hline & Ríos & 5 & 19 \\
\hline & Histórica & 5 & 19 \\
\hline & Etnografía & 2 & 8 \\
\hline & Restos prehistóricos & 1 & 4 \\
\hline & Manifestaciones religiosas & 1 & 4 \\
\hline & Arquitectura y espacios urbanos & 3 & 12 \\
\hline & Explotaciones industriales & 4 & 15 \\
\hline
\end{tabular}




\begin{tabular}{|c|c|c|c|}
\hline \multicolumn{4}{|c|}{ MATRIZ DE SISTEMATIZACIÓN DE ATRACTIVOS TURÍSTICOS } \\
\hline \multicolumn{4}{|c|}{ CLASIFICACIÓN } \\
\hline & CATEGORIZACIÓN & $\begin{array}{l}\text { CANTIDAD } \\
\text { DE } \\
\text { ATRACTIVO } \\
\text { S } \\
\end{array}$ & $\begin{array}{c}\text { PORCENTAJ } \\
\text { E }\end{array}$ \\
\hline \multicolumn{4}{|c|}{ SUBTIPO } \\
\hline & Laguna & 1 & 4 \\
\hline & Poza & 1 & 4 \\
\hline & Cordillera & 1 & 4 \\
\hline & Bosque húmedo occidental & 1 & 4 \\
\hline & Bosque montano bajo occidental & 1 & 4 \\
\hline & Rápidos o raduales & 1 & 4 \\
\hline & Cascadas & 4 & 15 \\
\hline & Arquitectura religiosa & 2 & 8 \\
\hline & Sitios arqueológicos & 1 & 4 \\
\hline & Arquitectura & 1 & 4 \\
\hline & Arquitectura civil & 1 & 4 \\
\hline & Grupo étnico kichwas de la sierra & 1 & 4 \\
\hline & Comidas y bebidas típicas & 1 & 4 \\
\hline & Fósiles petrificadas & 1 & 4 \\
\hline & Fiestas y celebraciones religiosas & 1 & 4 \\
\hline & Finca & 1 & 4 \\
\hline & Iglesia & 1 & 4 \\
\hline & Hacienda & 1 & 4 \\
\hline & Fábrica de quesos & 1 & 4 \\
\hline & Fábrica de panelas & 1 & 4 \\
\hline & Trapiche & 1 & 4 \\
\hline & Taller artesanal & 1 & 4 \\
\hline \multirow{2}{*}{$\begin{array}{c}\text { ESTADO DE } \\
\text { CONSERVACIÓ } \\
\mathrm{N} \\
\end{array}$} & NO ALTERADO & 17 & 65 \\
\hline & ALTERADO & 9 & 35 \\
\hline \multirow{3}{*}{$\begin{array}{c}\text { ESTADO DEL } \\
\text { ENTORNO }\end{array}$} & CONSERVADO & 21 & 81 \\
\hline & EN PROCESO DE DETERIORO & 5 & 19 \\
\hline & DETERIORADO & 0 & 0 \\
\hline \multirow{5}{*}{ JERARQUÍA } & $\mathrm{I}$ & 4 & 15 \\
\hline & II & 17 & 65 \\
\hline & III & 3 & 12 \\
\hline & IV & 0 & 0 \\
\hline & $\begin{array}{c}\text { SIN JERARQUIZACIÓN } \\
\text { (Manifestaciones culturales del } \\
\text { patrimonio intangible) }\end{array}$ & 3 & 12 \\
\hline
\end{tabular}

Los atractivos turísticos de mayor relevancia dentro de las zonas de intervención del MAGAP, se encuentran categorizados en 10 atractivos naturales y 16 culturales, el $62 \%$ se encuentran clasificados dentro de las manifestaciones culturales tangibles e intangibles, y el 38\% son sitios naturales. El $65 \%$ de los atractivos no se encuentran alterados en su estado de conservación y el $81 \%$ se encuentran conservados en su entorno. La jerarquía que más prevalece es la "II" con un $69 \%$, cabe mencionar que después del 
proceso de validación se encontró que 19 atractivos turísticos mantienen la jerarquía y 5 cambian porque se encuentran restaurados. Los atractivos naturales y culturales poseen un gran interés para la zona. Además los servicios básicos en las dos poblaciones se encuentran de la siguiente forma: El 78\% de las viviendas poseen Agua entubada, el 100\% servicio eléctrico, el 68\% servicio higiénico/ saneamiento, el 75\% servicio telefónico, y 48 carreteras de primer orden y $52 \%$ de segundo y tercer orden.

La planta turística existente en los cantones Alausí y Cumandá, muestran una cobertura en alojamiento del 56\%, en alimentación del $67 \%$, operación $50 \%$, recreación $60 \%$ y en el servicio de transporte un $58 \%$ en base a esta información se puede establecer que en los dos cantones, la planta turística es suficiente para impulsar Agroturismo, la mayoría de establecimientos de alojamiento y alimentación se encuentran en categorías III Y IV. Esto se resume en el siguiente cuadro.

Cuadro 4. Establecimientos turísticos

\begin{tabular}{|c|c|c|c|c|c|c|c|c|c|c|c|c|}
\hline \multicolumn{13}{|c|}{ ESTABLECIMIENTOS PRESTADORES DE SERVICIO TURÍSTICO } \\
\hline \multirow[t]{3}{*}{ Cantón } & \multicolumn{5}{|c|}{ ALOJAMIENTO } & \multicolumn{4}{|c|}{$\begin{array}{c}\text { ALIMENTACIÓ } \\
\mathrm{N} \\
\end{array}$} & $\begin{array}{l}\text { OPERACI } \\
\text { ÓN }\end{array}$ & $\begin{array}{l}\text { RECREACI } \\
\text { ÓN }\end{array}$ & $\begin{array}{c}\text { TRANSPO } \\
\text { RTE }\end{array}$ \\
\hline & \multicolumn{5}{|c|}{ CATEGORÍA } & \multicolumn{4}{|c|}{ CATEGORÍA } & CATEGO & CATEGORÍ & CATEGORÍ \\
\hline & I & $\begin{array}{l}\text { I } \\
\text { I }\end{array}$ & $\begin{array}{c}\text { II } \\
\text { I }\end{array}$ & $\begin{array}{l}\text { I } \\
\text { V }\end{array}$ & $\begin{array}{l}\text { CT } \\
\text { C }\end{array}$ & I & I & III & $\begin{array}{l}\text { I } \\
\text { V }\end{array}$ & Única & única & Única \\
\hline $\begin{array}{c}\text { Cuman } \\
\text { dá }\end{array}$ & 1 & 4 & $\begin{array}{l}1 \\
1\end{array}$ & & 4 & 13 & 5 & 18 & $\begin{array}{l}1 \\
3\end{array}$ & 2 & 3 & 14 \\
\hline Alausí & & & 4 & 2 & 3 & 4 & 2 & 6 & 3 & & 2 & 5 \\
\hline Total & 1 & 3 & $\begin{array}{l}1 \\
4\end{array}$ & 2 & 7 & 17 & 7 & 24 & $\begin{array}{l}1 \\
6\end{array}$ & 2 & 5 & 19 \\
\hline
\end{tabular}

\section{- Demanda}

Para el caso de la demanda una vez sistematizado los resultados se considera como perfil a las personas económicamente activas de las ciudades de Riobamba, Quito y Guayaquil, con un rango de edad entre los 18 a 35 años, de género masculino y femenino, con educación superior, que viajan acompañados, se dedican a trabajar en el sector publico y/o privado, tiene disponibilidad de tiempo de 1 a 2 días y están dispuestos a pagar entre 20 y 60 dólares por su viaje.

La demanda insatisfecha para el primer año es de 1762002, el modelo busca atender inicialmente al $3 \%$ esto es 529 personas. Esto debido a que es un producto nuevo y debe irse consolidando a través de diferentes estrategias. Para la demanda objetivo se diseñó 3 paquetes turísticos de con diferentes alternativas una ejemplo se presenta en el cuadro 5. 


\section{Diseño técnico de la ruta de agroturismo}

Cuadro 5. Ruta Agroturistica

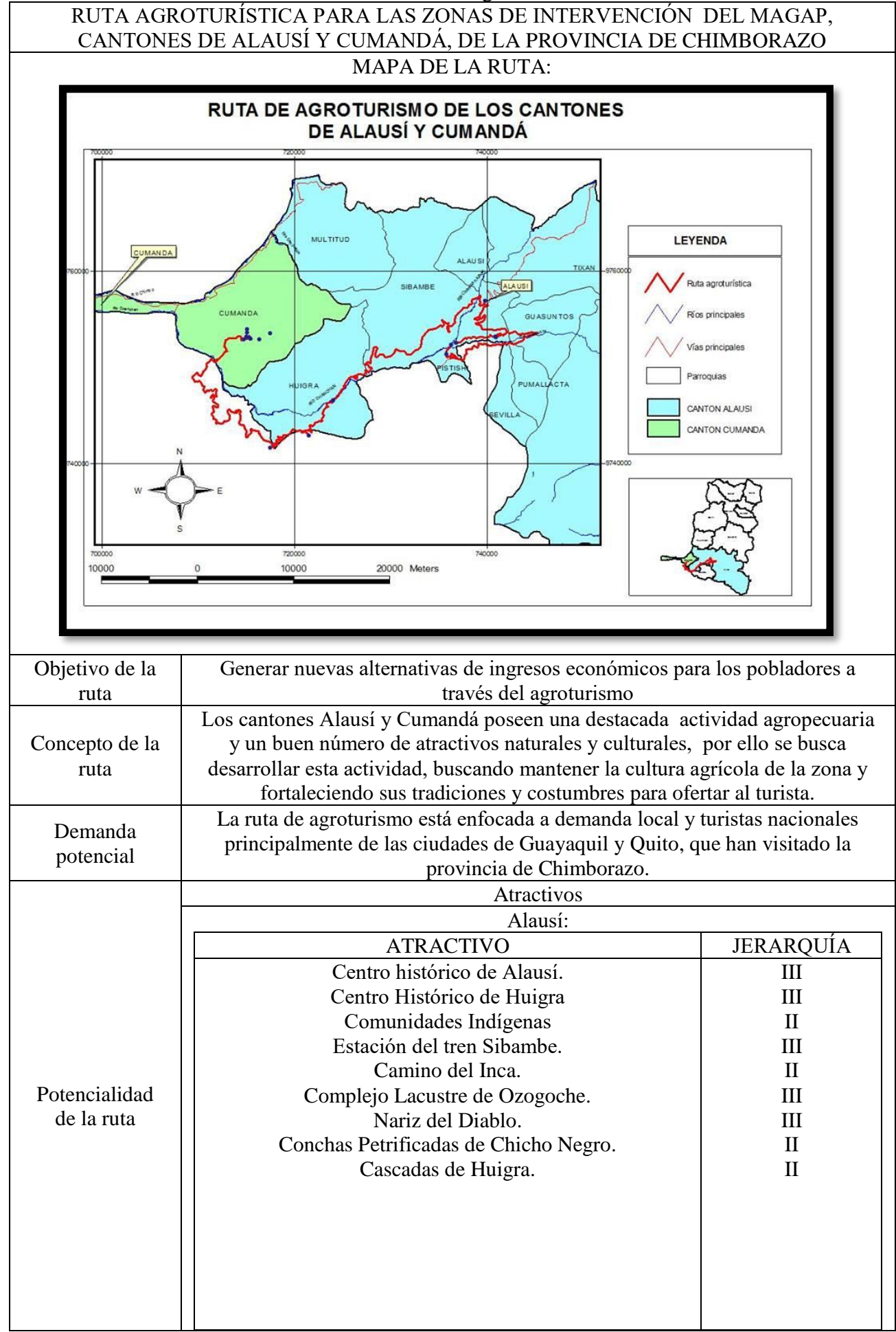




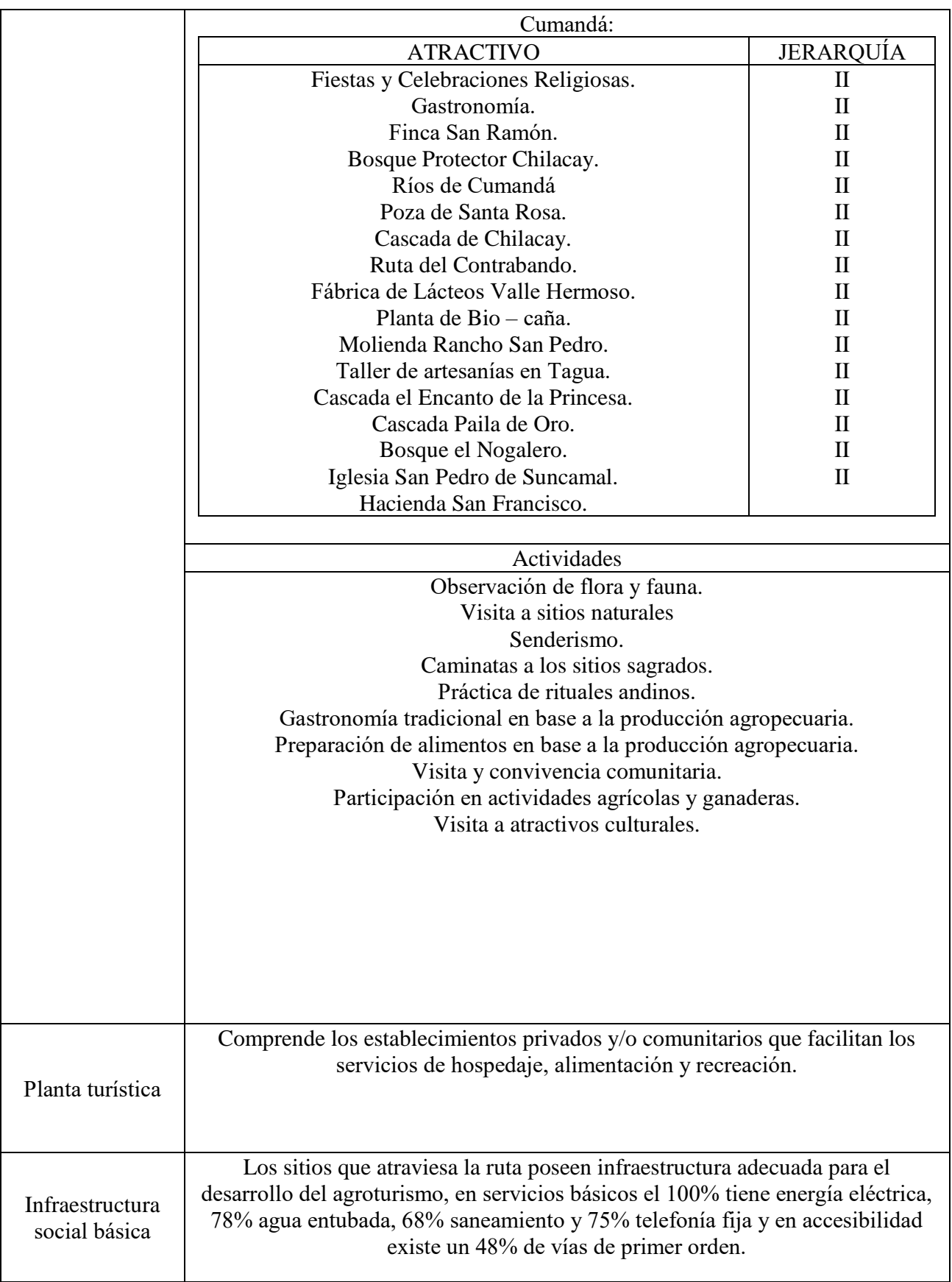




\section{a. Paquetes técnicos}

\begin{tabular}{|c|c|c|c|}
\hline \multicolumn{4}{|c|}{ PAQUETE No. 01 } \\
\hline \multicolumn{4}{|c|}{ Nombre del paquete: TRADICIÓN Y COSTUMBRE AGROPECUARIA } \\
\hline \multicolumn{3}{|c|}{ Código: MAGAP02 } & Estilo: Caminata \\
\hline \multicolumn{3}{|c|}{ Idioma de Guía: Español } & Duración: 4 días \\
\hline \multicolumn{3}{|c|}{ Recorrido: Alausí-Pistishi-Nizag-Huigra-Cumandá } & Carácter: Agroturístico - Descanso \\
\hline \multirow{2}{*}{\multicolumn{3}{|c|}{ Mercado Objetivo: Turismo Nacional }} & Grado de dificultad: Fácil \\
\hline & & & Segmento: (Jóvenes, adultos y adultos mayores) \\
\hline \multicolumn{4}{|c|}{ ITINERARIO } \\
\hline DÍAS & HORA & & ACTIVIDADES \\
\hline \multirow{11}{*}{ Día 1} & $07 \mathrm{~h} 00$ & Conce & ión y bienvenida Estación del Tren Alausí. \\
\hline & $07 \mathrm{~h} 30$ & & Salida a la parroquia Pistishi. \\
\hline & $08 \mathrm{~h} 15$ & Desayuno e & comedor turístico El Mirador parroquia Pistishi. \\
\hline & $09 \mathrm{~h} 30$ & Recorrido por & sembríos de aguacate, chirimoya y granja integral. \\
\hline & $11 \mathrm{~h} 00$ & & orrido por la Estación de Sibambe. \\
\hline & $12 \mathrm{~h} 30$ & Almuerzo e & comedor turístico El Mirador parroquia Pistishi. \\
\hline & $14 \mathrm{~h} 00$ & & Salida a la comunidad Nizag. \\
\hline & $14 \mathrm{~h} 40$ & & orrido por la comunidad de Nizag. \\
\hline & $18 \mathrm{~h} 00$ & Cena $\mathrm{e}$ & centro turístico de la comunidad de Nizag. \\
\hline & $19 \mathrm{~h} 00$ & & Noche cultural \\
\hline & $20 \mathrm{~h} 00$ & Descans & el centro turístico de la comunidad de Nizag. \\
\hline \multirow{9}{*}{ Día 2} & $06 \mathrm{~h} 00$ & & Salida al ordeño de leche. \\
\hline & $07 \mathrm{~h} 00$ & Des: & o con alimentos tradicionales de la zona. \\
\hline & $08 \mathrm{~h} 30$ & & olgata al mirador Cóndor Puñuna. \\
\hline & $11 \mathrm{~h} 00$ & & corrido por los huertos hortícolas. \\
\hline & $12 \mathrm{~h} 30$ & Almuerz & el centro turístico de la comunidad de Nizag. \\
\hline & $13 \mathrm{~h} 40$ & & isita al centro artesanal de Nizag. \\
\hline & $14 \mathrm{~h} 50$ & & Salida a Alausí. \\
\hline & $15 \mathrm{~h} 30$ & & nata por el centro histórico de Alausí \\
\hline & $19 \mathrm{~h} 00$ & & Cena. \\
\hline \multirow{9}{*}{ Día 3} & $07 \mathrm{~h} 00$ & & Desayuno \\
\hline & $08 \mathrm{~h} 00$ & & Salida a la parroquia de Huigra. \\
\hline & $09 \mathrm{~h} 00$ & & ata por las cascadas Panamá y Angas. \\
\hline & $12 \mathrm{~h} 30$ & & Almuerzo \\
\hline & $13 \mathrm{~h} 40$ & & Salida a Cumandá \\
\hline & $16 \mathrm{~h} 00$ & Recorrido & ráctica en la Molienda del Rancho San Pedro. \\
\hline & $16 \mathrm{~h} 30$ & & tación de un licor típico de Suncamal. \\
\hline & $18 \mathrm{~h} 30$ & & Cena en el recinto Suncamal. \\
\hline & $19 \mathrm{~h} 00$ & & Noche cultural y descanso. \\
\hline \multirow{9}{*}{ Día 4} & $07 \mathrm{~h} 00$ & & esayuno en el recinto Suncamal. \\
\hline & $08 \mathrm{~h} 30$ & & ita a la planta empacadora de orito. \\
\hline & $09 \mathrm{~h} 40$ & & a la fábrica de lácteos Valle Hermoso. \\
\hline & $11 \mathrm{~h} 00$ & & Visita a la planta Bio-caña \\
\hline & $13 \mathrm{~h} 00$ & & Almuerzo tradicional de la zona. \\
\hline & $14 \mathrm{~h} 30$ & Recol & por la iglesia de San Pedro de Suncamal. \\
\hline & $14 \mathrm{~h} 40$ & & ta y compra de artesanías de tagua. \\
\hline & $15 \mathrm{~h} 30$ & & ta por el bosque y cascada de Chilicay \\
\hline & $17 \mathrm{~h} 00$ & & Despedida. \\
\hline \multicolumn{4}{|c|}{ DESCRIPCIÓN DE PAQUETE } \\
\hline \multicolumn{2}{|c|}{ El paquete incluye } & Refr & $\begin{array}{l}\text { en base a productos propios de la zona. } \\
\text { Guía nativa } \\
\text { Material informativo }\end{array}$ \\
\hline \multicolumn{2}{|c|}{ El paquete no incluye } & & $\begin{array}{l}\text { Comidas y bebidas extras } \\
\text { Gastos personales } \\
\text { o no especificado en el programa }\end{array}$ \\
\hline \multicolumn{2}{|c|}{ Observaciones } & $\begin{array}{l}\text { El paquete es } \\
\text { productividac }\end{array}$ & $\begin{array}{l}\text { señado para turistas interesados en la naturaleza, } \\
\text { ícola, pecuaria y sobre todo la convivencia con la } \\
\text { gente. }\end{array}$ \\
\hline \multicolumn{2}{|c|}{ Requerimiento } & Doc & os personales para el registro de clientes. \\
\hline
\end{tabular}




\begin{tabular}{|c|c|}
\hline & $\begin{array}{c}\text { Ropa abrigada y cómoda para el frío y ropa liviana para el calor. } \\
\text { Protector solar. } \\
\text { Gafas, gorra. } \\
\text { Cámara fotográfica. }\end{array}$ \\
\hline Normas de comportamiento & $\begin{array}{c}\text { Otros que el cliente considere necesarios } \\
\text { Nespeto a la naturaleza y los grupos sociales } \\
\text { No botar basura }\end{array}$ \\
No sustraerse la flora y fauna de la zona.
\end{tabular}

\section{Esquematización del modelo Modelo de Canvas}

Como ya se mencionó la mejor forma de diseñar un modelo de negocio es a través de la aplicación del Canvas por ello el siguiente esquema explica el modelo en 9 pasos:

1. Segmento de mercado ¿Para quién estamos creando el valor? ¿Quiénes son nuestros clientes más importantes?

2. Relación con el cliente ¿Qué tipo de relación espera que establezcamos y mantengamos con cada uno de nuestros segmentos del cliente?

3. Canales de distribución ¿A través de que canales deseamos ser conectados? ¿Cómo se integran a nuestros canales?¿Cales funcionan mejor a menor costo?

4. Propuesta en valor ¿Qué valor entregamos al cliente?¿Que necesita y como satisfacemos al necesidad de nuestro cliente?

5. Actividades claves ¿Cuál es la actividad clave de nuestra empresa?¿Nuestra relación con el cliente?¿Cuál es la fuente de nuestros ingresos?

6. Recursos Claves ¿Qué recursos claves requiere nuestra propuesta?¿Nuestros canales de distribución?¿Relación con el cliente? ¿Fuentes de ingreso?

7. Socios claves ¿Quiénes son nuestros socios claves?¿Quiénes son nuestro proveedores?

8. Estructura de costos ¿Cuáles son los costos de nuestra empresa?¿Cuales son los recursos y actividades más costosas?

9. Fuentes de ingreso ¿Cuáles son nuestras fuentes de ingresos?¿Que vamos a vender? ¿Cuánto vamos a ganar?¿ Cómo vamos a cobrar? 
Figura 1. Modelo Canvas

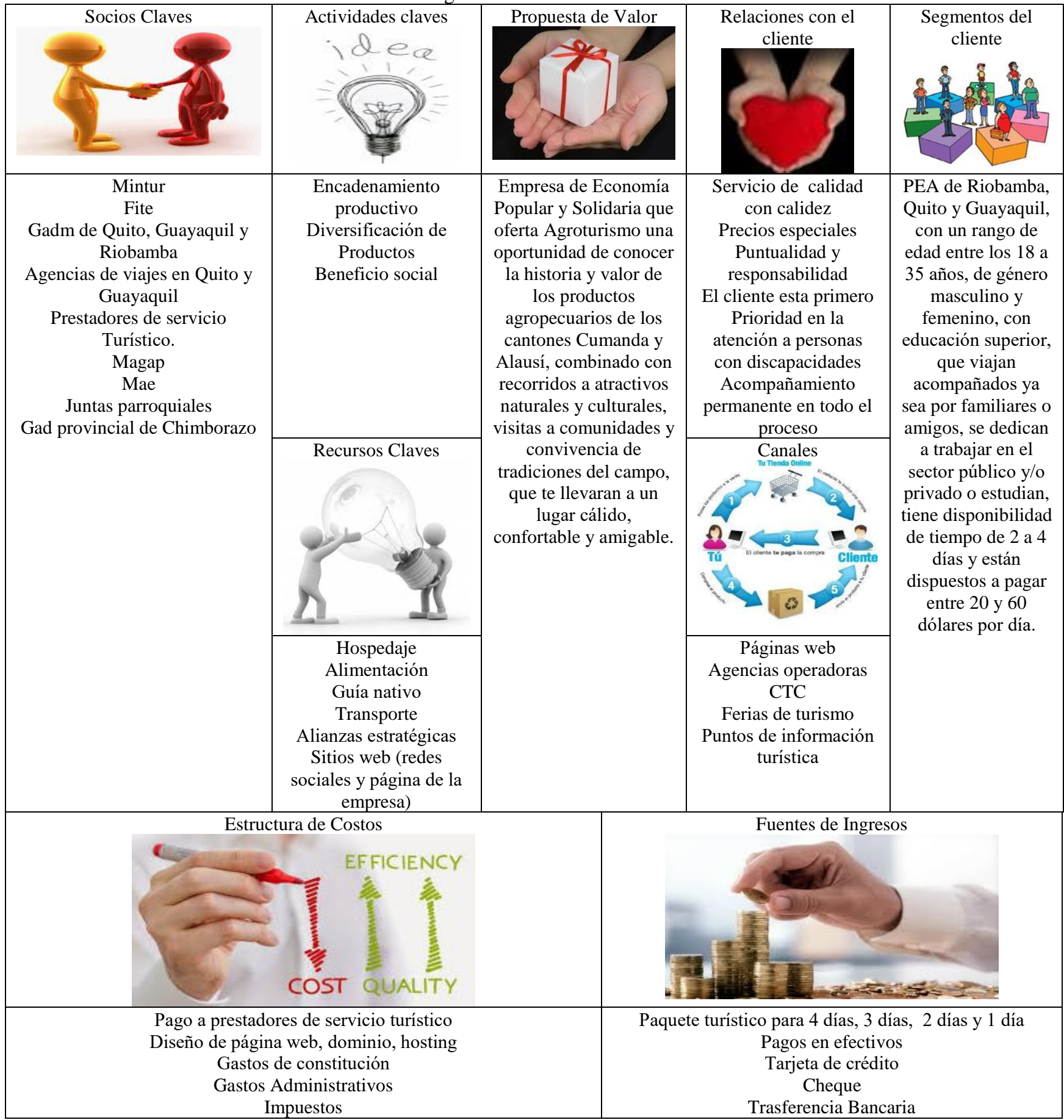




\section{Conclusion}

1. Se identificaron 33 productos agropecuarios en los cantones Alausí y Cumandá, considerados como principales e importantes en el desarrollo económico de la población. Estos sistemas de producción integran conocimientos, sabiduría, costumbres y tradiciones ancestrales, naturaleza, cultura, que generan un valor diferencial para la implementación de un producto agroturístico.

2. Para el desarrollo de la ruta de agroturismo se identificaron 26 atractivos naturales y culturales por donde se establece la ruta, la mayoría se encuentra en jerarquía II y III. En cada una de las zonas de intervención, la planta turística se encuentra apta para el desarrollo de la actividad agroturística. Se diseñó 4 rutas productos turísticos para 4, 3, 2 y 1 día.

3. El modelo Canvas esquematiza todos los componentes necesarios para diseñar un modelo de negocio factible, con claridad e identificar las debilidades del modelo y así las potencialidades del mismo con el fin de realizar un diseño más seguro y eficiente que proporcione mayor rentabilidad.

\section{References:}

1. Asociación de monitores medio ambientales Almijara. (2008). Turismo Sostenible. Recuperado el 13 de 08 de 2014, de http://www.turismo-sostenible.org/docs.php?did=1

2. Alegre, J, \& LLorenc, p. (2006). El Paquete Turístico de Todo Incluido: Un análisis de sus implicaciones económicas para el caso de las Islas Baleares. ., (pág. 34p.). Madrid.

3. Buesta, C (2015). Diseño de una ruta de Agroturismo en las zonas de intervención del Magap, cantones de Alausí y Cumandá de la provincia de Chimborazo. Espoch.

4. Consejo Cafetalero Nacional [COFENAC]. (31 de Enero de 2013). Situación del sector cafetalero ecuatoriano. 14.

5. Gobierno Parroquial San Jacinto del Búa. (2012). Plan de Desarrollo y Ordenamiento Territorial San Jacinto del Búa. San Jacinto del Búa.

6. Machado, E., \& Hernández, Y. (2009). Procedimiento para el Diseño de un Producto Turístico Integrado en Cuba. Recuperado el 12 de 09 de 2014, de www.teoríaypraxis.uqroo.mx

7. Magap \& Coordinación General del Sistema de Información Nacional. (2014). Boletín situacional café. 3-5.

8. Magap. (26 de Marzo de 2014). MAGAP fomenta la producción de café robusta en la costa.

9. Magap-Siagro. (2010). Compendio estadístico provincial año 2010Primer semestre.

10. Ministerio de Agricultura, Ganaderia, Acuacultura y Pesca [MAGAP]. (2012). Proyecto de Reactivación de la Caficultura Ecuatoriana. 
11. Ministerio de Agricultura, Ganadería, Acuacultura y Pesca [MAGAP]. (22 de julio de 2015). Entregan 100 mil chapolas de café robusta en Santo Domingo. Obtenido de http://www.agricultura.gob.ec/entregan100-mil-chapolas-de-cafe-robusta-en-santo-domingo/

12. Paspuel, W. (2015). La industria pide más café local. Revista Líderes.

13. Mintur (2007). Plan Nacional de Desarrollo Turístico - PLANDETUR 2020.

14. Mintur (2014). Barómetro Turístico. Recuperado el 15 de 08 de 2015, http://www.turismo.gob.ec/wpcontent/uploads/downloads/2013/02/PIMTE_2014.pdf

15. Prim, A. (12 de 2014). Innokabi. Recuperado el 28 de 07 de 2017, de Innokabi: http://innokabi.com/canvas-de-modelo-de-negocio/

16. Sistema Nacional de Información del MAGAP [SINAGAP]. (2016). Información de superficie, producción y rendimiento.

17. Fao. (2009). Consideraciones básicas sobre la agricultura sostenible. Recuperado el 15 de 08 de 2014, de http://maelac.files.wordpress.com/2009/09/agricultura-sostenibleconsideraciones-basicas-mayo-2009-acta-academica.pdf

18. Varela, M. (2012). Diseño de un producto turístico basado en los productos patrimoniales agrícolas de la provincia de Chimborazo. Tesis de grado. ESPOCH. RIOBAMBA. 